\title{
The Tangled Relationship Between Autoimmunity, Alzheimer's Disease, and Exercise
}

\author{
By Logan Townsend
}

Normally the immune system protects the body from potentially harmful substances by producing antibodies that destroy these substances. However, sometimes our immune system malfunctions and the body detects its own tissue as harmful substances and ultimately destroys healthy bodily tissues - this is known as autoimmunity. (1)

Alzheimer's disease (AD) is the most common form of dementia in the elderly and the fourth leading cause of death in Western countries. (2) It will be exceedingly difficult to pinpoint one thing responsible for the pathology of $A D$ (Rebecca MacPherson. Conversation with: Logan Townsend. 2015 Nov 11*), but evidence is beginning to emerge that autoimmunity may play a role. Autoimmunity in the pathogenesis of $A D$ has previously been ignored because antibodies capable of attacking neuronal cells circulate in healthy populations. However, the blood-brain barrier that was thought to protect the brain from systemic antibodies is dysfunctional in $A D$, conceivably making the brain susceptible to these circulating antibodies and thus contributing to the pathological features of AD. (1-3)

One of the neurological hallmarks of $A D$ is the accumulation of amyloid plaque that is detrimental to neuronal health. (4) Beta-site amyloid precursor protein cleaving enzyme 1 (BACE1) is important for the production of these plaques and can be used in early AD detection (4). The mechanisms behind the amyloid plaque formation and elevated BACE1 activity seen during $A D$ are complex, but increased and dysfunctional cellular stresses appear to be important precursors. (4) For instance, 5' AMP activated protein kinase (AMPK), the cellular energy and fuel gauge, is hyperactive in the brains of $A D$ afflicted individuals and is thought to be at least partially responsible for the accumulation of amyloid plaques and BACE1 activity in AD.

It is well known that exercise can prevent and treat obesity and glucose intolerance, both of which are significant risk factors for developing $A D$, and it now seems that exercise can also directly affect the neurological mechanisms of AD. Dr. Rebecca MacPherson, a post-doctoral fellow at the University of Guelph, investigated the effects of a single bout of exercise on markers of neurodegeneration in obese glucose intolerant mice. (4) This study demonstrated for the first time that a single bout of exercise ( 2 hours) reduced neuronal AMPK activity which could have neuroprotective effects since AMPK contributes to BACE1 activity and amyloid plaque formation. Indeed, "the most important finding from this study", according to Dr. MacPherson, "is that one bout of exercise can reduce BACE1 protein content and activity in the frontal cortex of obese glucose and insulin intolerant mice". These results highlight the therapeutic potential of a single bout of exercise to ameliorate changes in early $A D$-associated neuropathology.

At the same time, consistent exercise appears to rescue the cognitive declines and neuropathology of $A D$, regardless of disease progression or symptom severity. Cho and colleagues (5) introduced an exercise program of only 30 minutes of running 5 times a week for 12 weeks into a mouse model that demonstrates the cognitive deterioration and brain pathology of $A D$. Regardless of disease progression, the exercise intervention reversed cognitive declines and improved learning and memory. Moreover, extending on Dr. MacPherson's results, this study shows that prolonged exercise training (of 12 weeks) can significantly reduce 


\section{HealTh SCIENCE INQUiRY}

BACE1 proteins and reverse amyloid plaque accumulation.

(5)

As Dr. MacPherson notes, "BACE1 inhibition has become a key therapeutic target for Alzheimer's disease and there are currently several BACE1 inhibitors undergoing human clinical trials". Therefore, it is very promising that a single exercise session can reduce BACE1 content while consistent exercise training can similarly reduce BACE1 content in addition to plaque formation. $(4,5)$ Considering Canada's aging population, these studies are the first of many that will provide valuable information in terms of designing evidence-based preventative or therapeutic lifestyle interventions for AD.

\section{References}

1. D'Andrea M. Add Alzheimer's disease to the list of autoimmune diseases. Med Hypo. 2005; 64(3), 458-463.

2. Colasanti T, Barbati C, Rosano G, Malorni W, Ortona E. Autoantibodies in patients with Alzheimer's disease: pathogenetic role and potential use as biomarkers of disease progression. Autoimmu Rev. 2010; 9(12), 807-811.

3. Sardi F, Fassina L, Venturini L, Inguscio M, Guerriero F, Rolfo E, Ricevuti G. Alzheimer's disease, autoimmunity and inflammation. The good, the bad and the ugly. Autoimmu Rev. 2011; 11(2), 149-153.

4. MacPherson R E, Baumeister P, Peppler W T, Wright D C, Little J P. Reduced cortical BACE1 content with one bout of exercise is accompanied by declines in AMPK, AKT, and MAPK signaling in obese, glucose intolerant mice. J Appl Physiol. 2015; 119(10), 1097-1104.

5. Cho J, Shin M K, Kim D, Lee I, Kim S, Kang H. Treadmill running reverses cognitive declines due to Alzheimer's disease. Med Sci Sports Exerc. 2015; 47(9), 1814-1824.

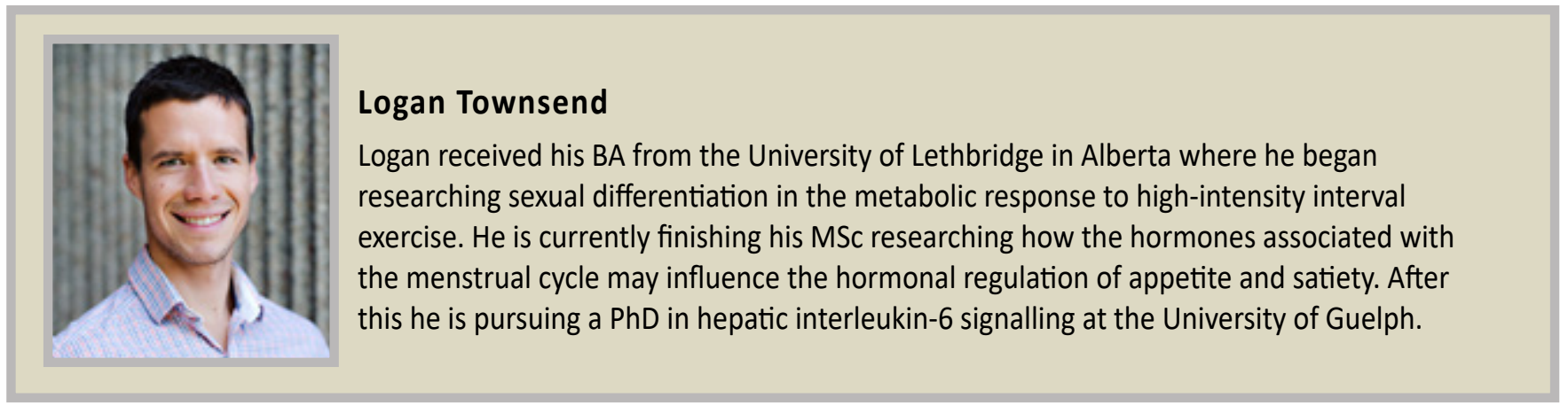

